\title{
Der Aufbau tief zerstörter Situationen
}

\section{Nachdem in meiner Praxis lange u.a. das ER-Stiftsystem zum Einsatz kam, machte mich der Komet-Fachberater Anfang die- sen Jahres auf den DentinPost X Coated (DPXCL6) aufmerksam. Ich begann - zunächst zögerlich - mit dem auffällig kurzen Stift mit dem großen Retentionskopf zu arbeiten. Inzwischen möchte ich den Stift nicht mehr missen. Damit können selbst tief zerstörte Situationen in kurzer Zeit effizient behandelt wer- den, wie der folgende Patientenfall zeigt.}

Der DentinPost X Coated ist in 2 Größen (090-rot und 070-orange) erhältlich. Zu beiden Größen steht der passende Erweiterer sowie das passende Aufrauinstrument zur Verfügung - ähnlich, wie man es vom ER-System mit Titanstiften kennt. In meiner Praxis kamen bisher fast ausschließlich die 070er Stifte zum Einsatz. Die Stifte haben eine Schaftlänge von nur 6 Millimetern, sodass die endodontisch aufbereitete Wurzel nicht so stark beansprucht wird, wie man es von Stiften mit längerem Schaft kennt, bei denen immer wieder Längsfrakturen der Wurzel zu beobachten sind. Der DentinPost X Coated hat am okklusalen Ende ein „Handlingsteil“, sozusagen einen kleinen Stift am Stift, der das Applizieren erleichtert und nach dem Einsetzen problemlos durch Abknicken entfernt wird.

Der neue Stift besitzt im Gegensatz zum DentinPost Coated einen sehr großen Retentionskopf, der es ermöglicht, auch tief zerstörte, endodontisch vorbehandelte

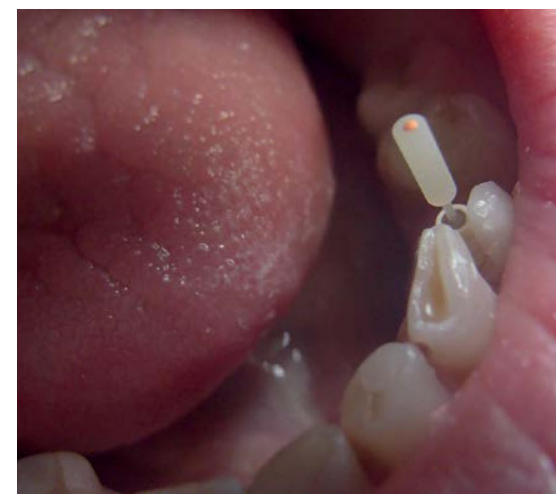

Abb. 2 Einprobe des Stifts.
Zähne wieder aufzubauen. Der Stift ist beschichtet. Durch die Silanisierung ist eine gute adhäsive Befestigung im vorbereiteten Wurzelkanal möglich.

Zum Befestigen eignen sich alle fließfähigen Kompositmaterialien. Letztendlich habe ich mich, nachdem ich einiges ausprobiert habe, für das Komposit-System DentinBond Evo/DentinBuild Evo entschieden, nicht zuletzt, um im Komet-System zu bleiben. Ich nutze beim Bonding die Single-Mix-Kapseln, denn das spart Zeit. Das Komposit kann zum Befestigen des Stifts und zum Aufbau des Stumpfs genutzt werden. Das Material ist sehr fest und formstabil. Wann immer möglich, arbeite ich mit Formkappen - das geht noch schneller und der Behandler erhält einen gut ausgeformten Stumpf. Das Material ist dualhärtend. Es härtet innerhalb von 3,5 min chemisch aus. Dabei ist darauf zu achten, dass sowohl der eingesetzte Stift als auch der Stumpf mit der Halogenlampe jeweils 20- $40 \mathrm{~s}$ gehärtet wird. Danach

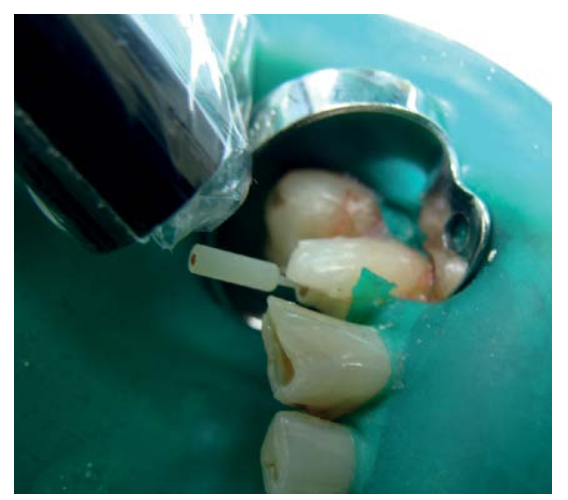

Abb. 3 Adhäsiv befestigter Stift.

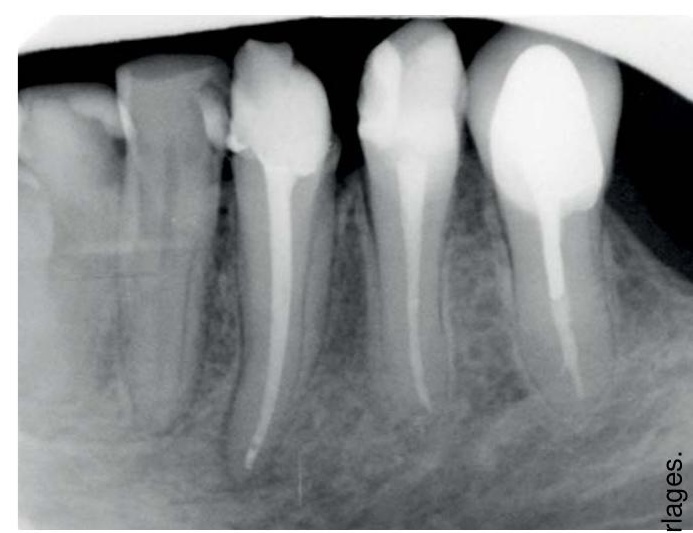

Abb. 1 Zahn 33 nach Wurzelfüllung.

kann der Stumpf mit Diamantschleifern und Hartmetallfinierern für die Restauration vorbereitet werden. Anfangs haben wir mit dem DentinPost X Coated nur im Frontzahngebiet gearbeitet. Inzwischen setzen wir die Stifte auch im Prämolarenund Molarenbereich ein.

\section{Patientenfall \\ $\nabla$}

Die Patientin (46 Jahre) stellte sich mit dem endodontisch noch nicht abgeschlossenen Zahn 33 vor. Nach erfolgter Wurzelfüllung wurde das weitere Vorgehen besprochen ( $\boldsymbol{O}$ Abb. 1). Die Patientin benötigt zwar herausnehmbaren Zahnersatz, der Restzahnbestand sollte aber möglichst erhalten bleiben. Der Zahn 33 war stark zerstört, nur die bukkale Wand stand noch zur Verfügung. Zur Stabilisierung des Zahns setzten wir den DentinPost X Coated ein. Nach Entfernung der Wurzelfüllung im Wurzelkanal mittels des Pilotbohrers 183LB aus dem ER-System, präparierte ich mit dem Kanalerweiterer 196D in 1 Schritt den Retentionskasten und das Stiftbett. Danach wurde die Wurzelkanalwand mit dem Aufrauinstrument 196DS bearbeitet (2- bis 3-mal drucklos rotieren). Der Stift wurde nach Einprobe und Alkoholdesinfektion im nach Vorschrift vorbereiteten Wurzelkanal adhäsiv befestigt ( $\odot$ Abb. 2 und 3): Unter Kofferdam auf die gereinigte, trockene Oberfläche einschließlich Wurzelkanäle DentinBond Evo auftragen, Überschuss aus den Kanälen mittels Papierspitze entfernen und $10 \mathrm{~s}$ mit Luftstrom leicht verblasen, danach 20 s lichthärten. DentinBuild Evo auf den Stift auftragen, Stift unter leicht drehender Bewegung in den vor- 


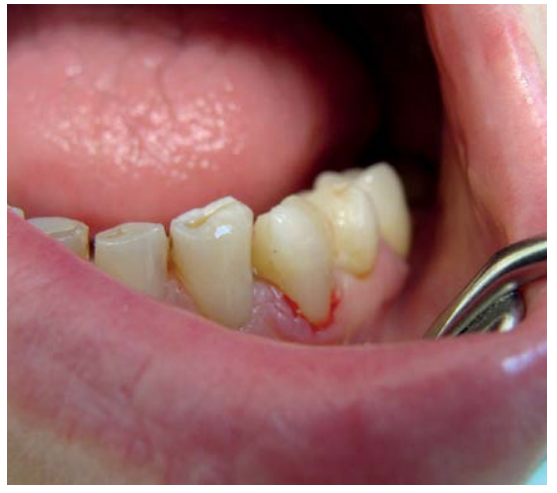

Abb. 4 Rekonstruierter Zahn 33.

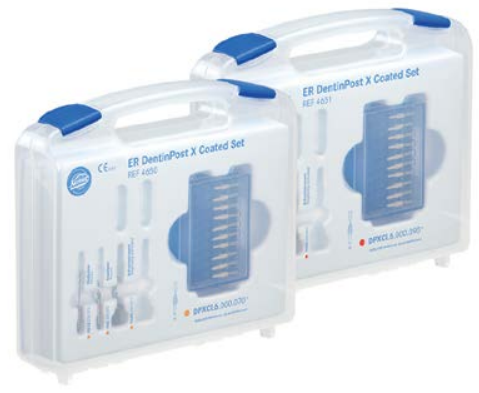

Abb. 5 a Die Sets 4650 und 4651 für den Einstieg: 10 Stifte plus passendes Instrumentarium. b Die Instrumente in entsprechender Reihenfolge - vom Pilotbohrer bis zum Stift. bereiteten Kanal einbringen, dann $20 \mathrm{~s}$ lichthärten, um den Stift zu fixieren. Das Handlingsteil wurde anschließend entfernt. Um den Stumpf wurde mittels Matrize zunächst Dentin Build Evo appliziert, das 40 s ausgehärtet wurde, anschließend baute ich mit einem farblich passenden Komposit den Zahn wieder auf. In der gleichen Sitzung wurde Zahn 32 mit einer Komposit-Füllung versorgt. Die gesamte Sitzung dauerte ca. 30 min und die Patientin konnte mit einem rekonstruierten Zahn 33 die Praxis verlassen ( 0 Abb. 4).

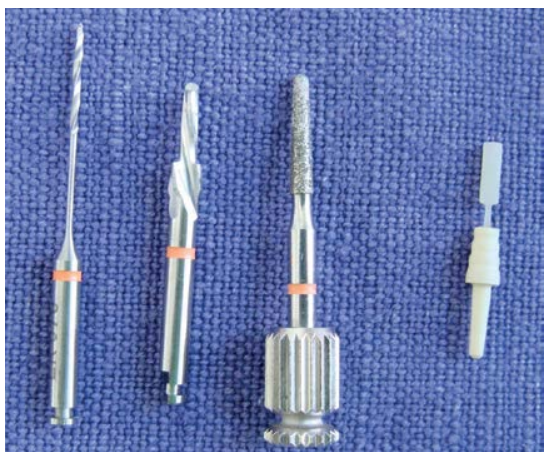

Die Präparation des Zahns zur Aufnahme einer Krone ist möglich.

\section{Resümee}

$\nabla$

Den großen Vorteil des DentinPost X Coated sehe ich in der Kürze des Stifts. Er schont die Wurzel. Der Stift ist sehr gut adhäsiv zu befestigen und sein großer Retentionskopf erlaubt es, auch bei tief zerstörten Zähnen eine Rekonstruktion zu wagen - und das bei vergleichsweise geringem Zeitaufwand und zu einem erschwinglichen Preis. Die Stifte werden je Größe in einem Intro-Set zusammen mit den passenden Instrumenten angeboten und können anschließend im 10er-Pack nachbestellt werden ( $\bullet$ Abb. 5). Ich empfehle, hin und wieder das gesamte Set neu zu erwerben, um wieder scharfe Instrumente zu erhalten. Wir haben seit Beginn des Jahres wohl fast die 4. 10er-Packung aufgebraucht! Durch die einfache Anwendung kann auch Patienten im Notdienst, die sich mit frakturierten Zähnen bzw. Kronen vorstellen, wunderbar geholfen werden.

Kontakt

Dipl. Stom. Karin Eschert

Karl-Ernst-Str. 8

99887 Georgenthal

k.eschert@dental-power.de 